\title{
Extreme value predictions using FORM for ship roll motions
}

\author{
Choi, Ju Hyuck; Jensen, Jørgen Juncher
}

\section{Published in:}

Marine Structures

Link to article, DOI:

10.1016/j.marstruc.2019.03.001

Publication date:

2019

Document Version

Peer reviewed version

Link back to DTU Orbit

Citation (APA):

Choi, J. H., \& Jensen, J. J. (2019). Extreme value predictions using FORM for ship roll motions. Marine Structures, 66, 52-65. https://doi.org/10.1016/j.marstruc.2019.03.001

\section{General rights}

Copyright and moral rights for the publications made accessible in the public portal are retained by the authors and/or other copyright owners and it is a condition of accessing publications that users recognise and abide by the legal requirements associated with these rights.

- Users may download and print one copy of any publication from the public portal for the purpose of private study or research.

- You may not further distribute the material or use it for any profit-making activity or commercial gain

- You may freely distribute the URL identifying the publication in the public portal

If you believe that this document breaches copyright please contact us providing details, and we will remove access to the work immediately and investigate your claim. 


\title{
Extreme value predictions using FORM for ship roll motions
}

\author{
Ju-hyuck Choi and Jørgen J uncher J ensen \\ Department of Mechanical Engineering, Technical University of Denmark
}

\section{Abstract}

Extreme value predictions of the roll motion of ships are considered within the framework of the First Order Reliability Method focusing on the applicability of the Poisson assumption for the peak value statistics. The examples considered are a ship rolling in dead sea condition under the combined action of waves and wind and a ship experiences parametric rolling.

Keywords. Extreme value prediction, FORM, up-crossing rate, Poisson assumption, rolling in dead ship condition, parametric rolling

\section{I ntroduction}

The International Maritime Organization (IMO) is currently developing Second Generation Intact Stability Criteria for ships, see, e.g. Tompuri et al. (2015). These completely revised rules include the possibility to account for the dynamics of ships using time-domain simulations of the roll motion under different operational conditions, considering different failure scenarios (pure loss of stability, parametric roll, dead ship, excessive acceleration and surf riding/broaching) and involve different levels of complexity and corresponding accuracy. Hence, it is important to derive/suggest effective statistical methods for extreme value prediction for ship roll motion.

The Poisson assumption is often used for extreme value prediction for stationary stochastic processes and requires only two parameters: the up-crossing rate and the time duration of the response. For a linear system, the up-crossing rate is a product of the mean zero up-crossing rate and an exponential term depending on the threshold level in terms of the reliability index. For non-linear systems, the up-crossing rate has a more complicated form, and moreover, the reliability index is not a linear function of the threshold level. The question is then to determine the reliability index and an accurate model of the up-crossing rate for non-linear systems. The First Order Reliability Method (FORM) can be used to determine the reliability index and it has been shown in several papers that often a good agreement is found as compared to direct Monte Carlo Simulations (MCS), e.g. Der Kiureghian (2000), Jensen (2015), Choi et al. (2017) and Jensen et al. (2017). The reason is that the reliability index is calculated using the true non-linear limit state function. Further, the up-crossing rate for non-linear systems can be simplified by considering that the mean zero up-crossing rate depends on the threshold level and is thus not a real zero up-crossing rate, but still a measure of the time between up-crossing. The mean zero up-crossing rate can be estimated from the linearized FORM solution, Jensen and Capul (2006), Fujimura and Der Kiureghian (2007), Garrè and Der Kiureghian (2010). However, due to the 
linearization it might not be as accurate as the reliability index, especially for systems with very strong (or bifurcation type of) non-linearity.

Hence, this paper attempts to improve the estimation of the up-crossing rate for non-linear response based on the framework of the First Order Reliability Method. Two different cases of large roll motions of ships are considered. One is rolling in beam seas, where the non-linearity slowly increases with roll angle in the reliability index curve, and the other is parametric rolling in longitudinal seas, which is mainly a pure non-linear system without any linear components in the reliability index curve.

In addition to FORM, the Second Order Reliability Method (SORM) is applied in the calculation of the reliability index. Compared to the FORM results, SORM does not provide a noticeable improvement in the calculation of the reliability index to the present examples.

The mean zero up-crossing rate from the linearized FORM solution is modified for bandwidth and clump clustering effect using Vanmarcke formula, Vanmarcke (1975). An investigation is conducted using a numerical method to check the feasibility of applying the Vanmarcke's factor to the present non-linear responses. From the statistical analysis it is found that by applying the factor, the dependency between the peaks can be determined with reasonable accuracy for both linear and non-linear responses. However, for the extreme values, the correction seems not necessary as the dependency between the peaks are negligible.

\section{First Order Reliability Method}

A straightforward procedure for solving non-linear problems formulated in the time-domain is to apply direct Monte Carlo Simulations, but usually the computational effort needed for extreme value predictions is prohibitively large. An alternative is to look at approximate procedures, which focuses on the tail in the probability distribution. A good candidate is the First Order Reliability Method (FORM), widely used in structural reliability analyses, but also an efficient method for extreme value predictions, as suggested originally by Der Kiureghian (2000) and derived for wave loads by Jensen and Capul (2006). A short outline of the FORM procedure can be found in Jensen (2015), and part of it is recapitulated in this section. The starting point is a limit state surface $G$ defined as:

$$
G(\underline{u}) \equiv x_{0}-X(0 \mid \underline{u})=0
$$

where $\underline{u}^{T}=\left[u_{1}, u_{2}, \ldots, u_{n}\right]$ are uncorrelated standard normal distributed variables defining the stochastic variations of the input $Y(t \mid \underline{u})$, e.g. the wave elevation and gust wind speed. Realizations of the associated wave response $X(t \mid \underline{u})$ are determined by a time domain analysis 
covering the time range $-t_{0} \leq t \leq 0$. For a stationary stochastic process the value of $t_{0}$ is unimportant as long as it is longer than the memory in the system, i.e. the influence at $t=0$ of the initial conditions at $t=-t_{0}$ is negligible. The realisation, which reaches the given threshold (peak) $x_{0}$ at $t=0$ with the highest probability, is sought. The corresponding values $\underline{u}^{*}$ of $\underline{u}$ is denoted the design point and uniquely defines the deterministic input process $Y\left(t \mid \underline{u}^{*}\right)$ which, due to the normal distributions of $\underline{u}$, also is the most probable input process leading to the desired response $x_{0}$ at $t=0$. Hence, it can be considered as the best choice for a deterministic critical input process, e.g. a critical wave episode.

The value of $\underline{u}^{*}$ is the solution to the non-linear optimization problem:

$$
\underline{u}^{*}: \text { Minimize } \underline{u}^{T} \underline{u} \text {; Subject to } G(\underline{u})=0
$$

The design point $\underline{u}^{*}$ is found using the real non-linear limit state function. Therefore, the probability $P\left(X(0)>x_{0}\right)=P(G<0)$ cannot in general be calculated as it depends on the nonlinearity in the response $X(t \mid \underline{u})$. Instead, it is in the FORM approach approximated by the probability of exceedance of the linearized limit state surface function $G_{F O R M}(\underline{u})$, obtained by linearizing $G(\underline{u})$ around the design point:

$$
G(\underline{u}) \simeq G_{F O R M}(\underline{u})=G\left(\underline{u}^{*}\right)+\left(\left.\frac{\partial G}{\partial \underline{u}}\right|_{\underline{u}=\underline{u}^{*}}\right)^{T}\left(\underline{u}-\underline{u}^{*}\right)=0
$$

The design point vector $\underline{u}^{*}$ is normal to the linearized limit state surface and measures the shortest distance from the origin to the limit state surface.

The normal vector $\underline{e}$ is given as

$$
\underline{e}=\left.\frac{\partial X}{\partial \underline{u}}\right|_{\underline{u}=\underline{u}^{*}}=\left[e_{1}, e_{2}, \ldots, e_{n}\right]^{T} ; \quad \underline{e}^{T} \underline{e} \equiv\|\underline{e}\|^{2}
$$

As $G\left(\underline{u}^{*}\right)=0$ according to Eq. (2) the linearized limit state surface, Eq. (3) can be written

$$
\underline{e}^{T} \underline{u}^{*}-\underline{e}^{T} \underline{u}=0
$$


Because the linearization is around a design point usually associated with a low probability of occurrence, this linearization has been denoted Tail-equivalent linearization, Fujimura and Der Kiureghian (2007).

The linearized response $X(0)$ is a stationary Gaussian process with variance

$$
m_{0}=E\left[X^{2}(0)\right] \simeq E\left[\left(\underline{e}^{T} \underline{u}\right)^{2}\right]=\underline{e}^{T} \underline{e} \equiv\|\underline{e}\|^{2}
$$

and, hence

$$
P\left(X(0)>x_{0}\right) \simeq P\left(\underline{e}^{T} \underline{u}>\underline{e}^{T} \underline{u}^{*}\right)=\Phi(-\beta)
$$

Here $\beta$ is the reliability index, defined as shortest distance to the limit state surface:

$$
\beta=\sqrt{\underline{u}^{* T} \underline{u}^{*}}=\frac{\underline{e}^{T} \underline{u}^{*}}{\|e\|}
$$

Also, from geometrical considerations

$$
\underline{u}^{*}=\frac{\beta}{\|e\|} \underline{e}
$$

For a large value of $\beta$ the linearization of the limit state function, Eq. (3), does not change the probability of exceedance $P\left(X(0)>x_{0}\right)$ much and hence the FORM approximation, Eq. (7), is generally rather accurate as illustrated by comparison with Monte Carlo Simulations in e.g. in Choi et al. (2017) and Jensen et al. (2017) for ship intact stability problems.

The Second Order Reliability Method (SORM) can in many cases yield an improvement in the FORM results. The basic principle is to make a quadratic approximation to the limit state function $G_{F O R M}(\underline{u})$ at the FORM design point $\underline{u}^{*}$. Various SORM formulations exist, see e.g. Köylüoglu and Nielsen (1994), but in the present paper the original exact asymptotic formulation due to Breitung (1984) has been used together with the effective computational procedure described in Breitung (2015). The SORM results is presented by the reliability index $\beta_{\text {SORM }}$

$$
\beta_{\text {SORM }}=-\Phi^{-1}(C \Phi(-\beta))
$$

where $C$ is the SORM correction factor, Breitung (1984). It is noted that $C$ depends on the Hessian matrix for the limit state function at the design point and thus a much larger accuracy is often needed in the SORM calculations than for the FORM results alone. 


\section{Extreme value predictions}

For extreme value estimations of linear systems, the mean up-crossing rate

$$
v^{+}\left(x_{0}\right)=\frac{1}{2 \pi} \sqrt{\frac{m_{2}}{m_{0}}} \exp \left(-\frac{\left(x_{0}-x_{\text {mean }}\right)^{2}}{2 m_{0}}\right)
$$

is very useful. It requires calculation of the spectral moment $m_{2}$ and therefore also a spectral formulation of the response. As shown in Jensen (2011) the spectral density $S_{x}(\omega)$ of the linearized response becomes

$$
S_{x}\left(\omega_{i}\right) d \omega=\left(\left.\frac{\partial X}{\partial u_{i}}\right|_{\underline{u}=\underline{u}^{*}}\right)^{2}=e_{i}^{2}
$$

when each $u_{i}$ is associated with a sinusoidal linearized response variation in time with frequency $\omega_{i}$. Hence,

$$
\begin{aligned}
& m_{0}=\int S_{x}(\omega) d \omega=\sum_{i=1}^{n} e_{i}^{2}=\|e\|^{2} \\
& m_{2}=\int \omega^{2} S_{x}(\omega) d \omega=\sum_{i=1}^{n} \omega_{i}^{2} e_{i}^{2}
\end{aligned}
$$

It is noted that both $m_{0}$ and $m_{2}$ depend on the design point and thereby on $x_{0}$. Furthermore,

$$
x_{\text {mean }}=X\left(\underline{u}^{*}\right)-\underline{e}^{T} \underline{u}^{*}=x_{0}-\underline{e}^{T} \underline{u}^{*}
$$

Eq. (11) can then be written, using Eqs. (8), (13) and (14)

$$
v^{+}\left(x_{0}\right)=\frac{1}{2 \pi \beta} \sqrt{\sum_{i=1}^{n} \omega_{i}^{2} u_{i}^{* 2}} \exp \left(-\frac{\beta^{2}}{2}\right)
$$

The autocorrelation function of the linearized FORM solution becomes

$$
R\left(t \mid \underline{u}^{*}\right)=\int S_{x}(\omega) \cos \omega t d \omega=\sum_{i=1}^{n} e_{i}^{2} \cos \omega_{i} t
$$

The corresponding most probable linear response is then 


$$
X\left(t \mid \underline{u}^{*}\right)_{\text {Linear }}=\frac{x_{0}}{m_{0}} R\left(t \mid \underline{u}^{*}\right)
$$

A comparison between $X\left(t \mid \underline{u}^{*}\right)_{\text {Linear }}$ and $X\left(t \mid \underline{u}^{*}\right)$ provides an indication of the how close the FORM linearization is to the real limit state surface $G(\underline{u})$ around the design point $\underline{u}^{*}$.

Extreme value statistics can often be based on the assumption of independent and identically distributed peaks (i.i.d. process) implying a Poisson model. For stationary processes the probability of exceedance the level $x_{0}$ during the time $T$ can then be written

$$
P\left[\max _{T} x(t)>x_{0}\right]=1-\exp \left(-v^{+}\left(x_{0}\right) T\right)
$$

The assumption of independent peaks usually holds for large threshold values $x_{0}$ provided the response is not too narrow-banded. To account for a possible correlation between adjacent peaks and grouping effects various procedures exist, see e.g. Naess et al. (2010) and Garrè and Der Kiureghian (2010). Often the correction due to Vanmarcke (1975) based on envelope crossings is applied. In the present FORM formulation, where the linearization around the design point results in a Gaussian process, the Vanmarcke correction factor to the mean up-crossing rate, Eq. (11), can be written

$$
c_{v}\left(x_{0}\right)=\frac{1-\exp \left(-\sqrt{2 \pi} q^{1.2} \beta\right)}{1-\exp \left(-0.5 \beta^{2}\right)}
$$

Here $q$ is a bandwidth factor:

$$
q\left(x_{0}\right)=\sqrt{1-\frac{m_{1}^{2}}{m_{0} m_{2}}}=\sqrt{1-\frac{\left.\left(\sum_{i=1}^{n} \omega_{i} u_{i}^{* 2}\right)\right)^{2}}{\beta^{2} \sum_{i=1}^{n} \omega_{i}^{2} u_{i}^{* 2}}}
$$

The exponent 1.2 on $q$ in Eq. (19) is purely emperical, suggested in Vanmarcke (1975), to account for clump clustering effects. The probability of exceedance is then, Vanmarcke (1975)

$$
P\left[\max _{T} x(t)>x_{0}\right]=1-\left(1-\exp \left(-0.5 \beta^{2}\right)\right) \exp \left(-c_{V} v^{+}\left(x_{0}\right) T\right) \simeq 1-\exp \left(-c_{v} v^{+}\left(x_{0}\right) T\right)
$$

Introducing

$$
T_{0}\left(x_{0}\right)=\frac{e^{-0.5 \beta^{2}}}{v^{+}\left(x_{0}\right)} ; \quad T_{0, \text { corr }}\left(x_{0}\right)=\frac{T_{0}\left(x_{0}\right)}{c_{v}\left(x_{0}\right)}
$$


Eq. (21) can be written in the usual format of the Poisson model:

$$
P\left[\max _{T} x(t)>x_{0}\right]=1-\exp \left(-T / T_{0, \text { corr }}\left(x_{0}\right) \exp \left(-0.5 \beta\left(x_{0}\right)^{2}\right)\right)
$$

It should be emphasized that contrary to the standard Poisson model for linear systems, the 'mean zero-upcrossing' period $T_{0, \text { corr }}$ is now a function of the threshold value $x_{0}$ and thus different results are obtained for different threshold values. In the Poisson model, Eq. (23), $T_{0, \text { corr }}\left(x_{0}\right)$ represents conceptually the mean period between independent peaks in the linearized response with bandwidth correction, but is actually just a normalization factor depending on the threshold level.

\section{Roll motions of ships with a simplified model}

In the following two examples are considered: a relative broad-banded case (ship rolling in beam sea) and a very narrow-banded case (parametric rolling of a ship).

\section{Ship rolling in beam seas}

A fictitious Panamax container ship without forward speed in beam seas is considered. The ship parameters (length $284.72 \mathrm{~m}$, breadth $32.2 \mathrm{~m}$, draft $10.5 \mathrm{~m}$ ) and its operational condition (significant wave height $11 \mathrm{~m}$, zero-crossing wave period 12s) are the same as in Choi et al. (2017) for their first example. Fig. 1 shows the GZ curve for the Panamax container ship and the discretization of the wave elevation and gust wind speed spectra are shown in Fig. 2 . The results from the FORM and Monte Carlo Simulation (MCS) analyses in terms of the reliability index $\beta$ are given in Fig. 3, taken from Choi et al. (2017). In addition new SORM results are included. A rather good agreement between FORM and MCS is seen and also the ability of FORM to cover roll angles up to capsize ( $>43$ deg.) whereas the SORM results from the applied SORM procedure differ significantly from the MCS and also shows an un-physical decrease at capsize. The reliability index from the FORM analysis will therefore be used in the extreme value calculations.

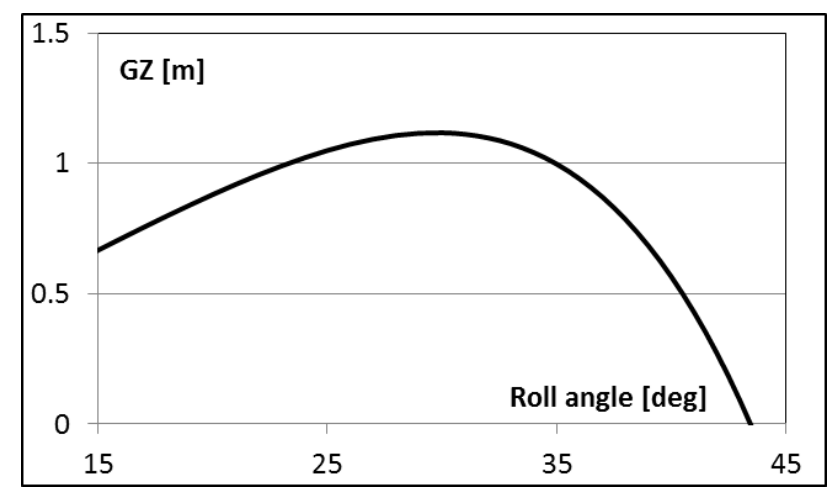

Fig. 1. GZ curve for the vessel, $G M=2.5 \mathrm{~m}$

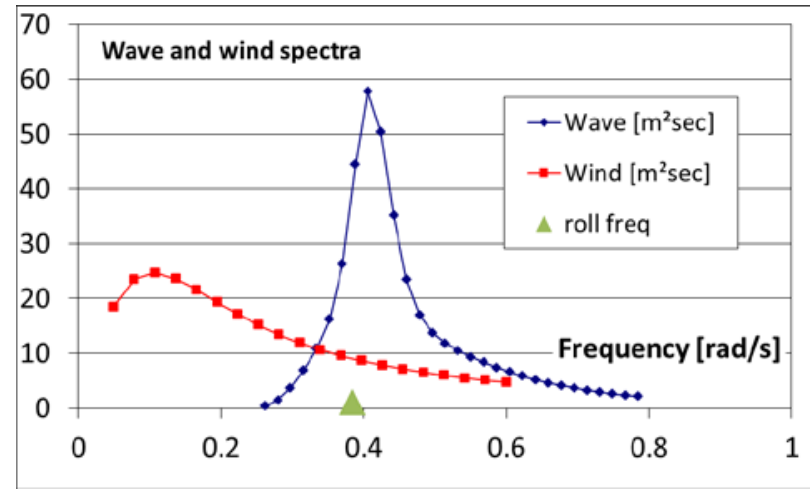

Fig. 2. Wave elevation and gust wind speed spectra 
In the following the application of the Poisson model, Eq. (23), is discussed. The bandwidth factor $q$, Eq. (20), and the Vanmarcke correction factor $c_{v}$, Eq. (19) are shown in Fig. 4 together with the ratio between $T_{0, \text { corr }}$ and $T_{0}$ are given. $T_{0}$ does not depend significantly on the target angle and thus the variation in $T_{0 \text {,corr }}$ with target angle is nearly solely due to the Vanmarcke correction, Eq. (19) and not the FORM linearization.

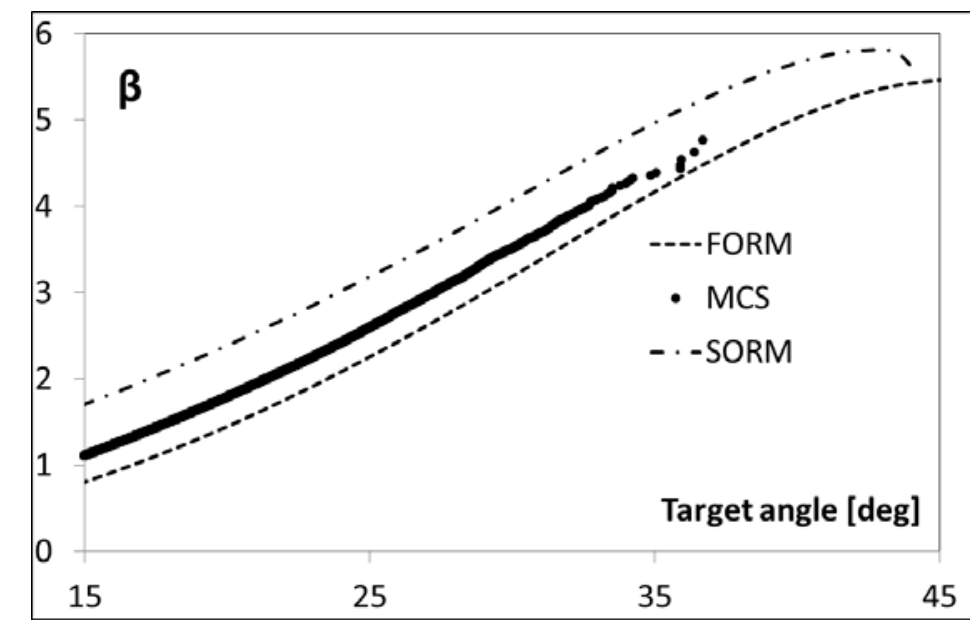

Fig. 3. Reliability index $\beta$ from FORM, SORM and MCS, beam sea condition

Fig. 4 shows that $c_{v}$ is close to one for peaks larger than about 35 deg., i.e. close to capsize. For small roll angles a rather strong correlation between adjacent peaks is found, where $T_{0, \text { corr }}$ is nearly twice the value of uncorrected value $T_{0}$ for small roll angles. For roll angle above 30 deg., the mean time between independent peaks is about 15-17 sec., which is close to the roll natural period $T_{\text {roll }}=16.5 \mathrm{sec}$. of the vessel, as determined using the initial GM value. Hence, extreme value predictions can be performed using Eq. (23) with $T_{0, \text { corr }}$ replaced by $T_{\text {roll }}$ without notable error. 


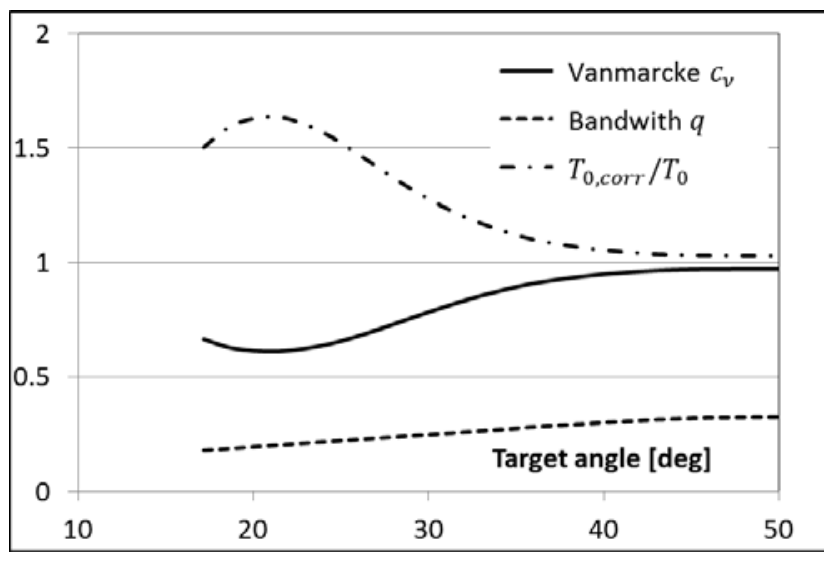

Fig. 4. Bandwidth factor $q$ and Vanmarcke factor $c_{v}$ and the ratio $T_{0, \text { corr }} / T_{0}$, beam sea condition

$$
\text { ( } T_{\text {roll }}=16.5 \mathrm{sec} \text {., } T_{0} \approx 15 \sim 17 \mathrm{sec} \text {.) }
$$

The FORM analysis also yields the most probable wave and response scenario leading to a given target roll angle. An example is given in Fig. 5, taken from Choi et al. (2017). The target roll angle is $37 \mathrm{deg}$. and the time between the target angle and the nearest preceding peak is 16.5 sec., i.e. very close to findings above. This most probable response scenario is compared in the figure with the linearized most probable response, Eq. (17), and a good agreement is seen, indicating that the FORM linearization around the design is rather accurate at this target angle. Fig. 6 shows the linearized roll spectrum, Eq. (12), for the target angle 37 deg. The two contributions from waves and gust wind make the total spectrum rather broad banded and hence the adjacent peaks are nearly uncorrelated as also indicated by the Vanmarcke correction factor $c_{v}$ being close to one at 37 deg.

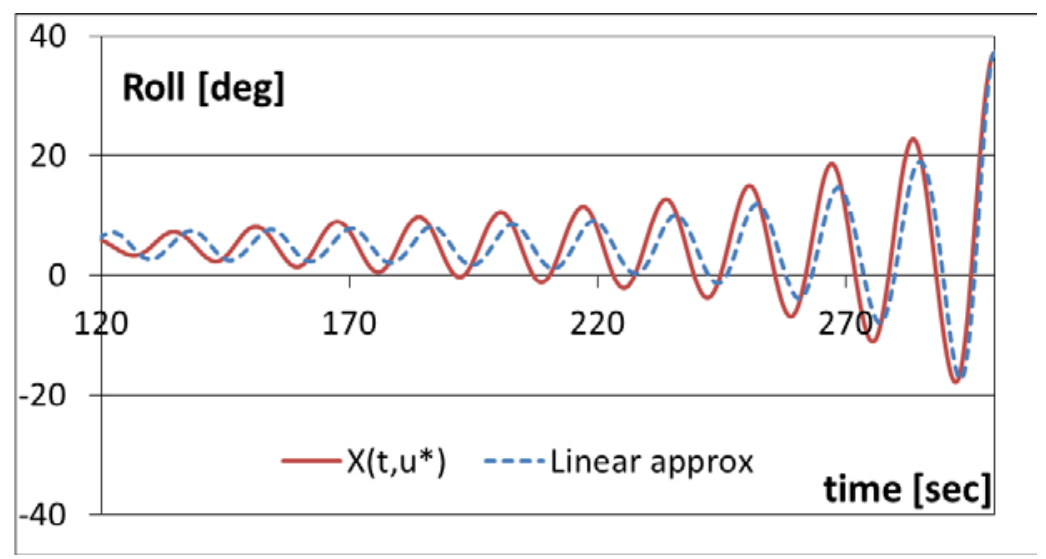

Fig. 5. Most probable dead ship rolling scenario $X\left(t \mid \underline{u}^{*}\right)$ and the linear approximation, Eq. (17). Target roll angle 37 deg., beam sea condition 


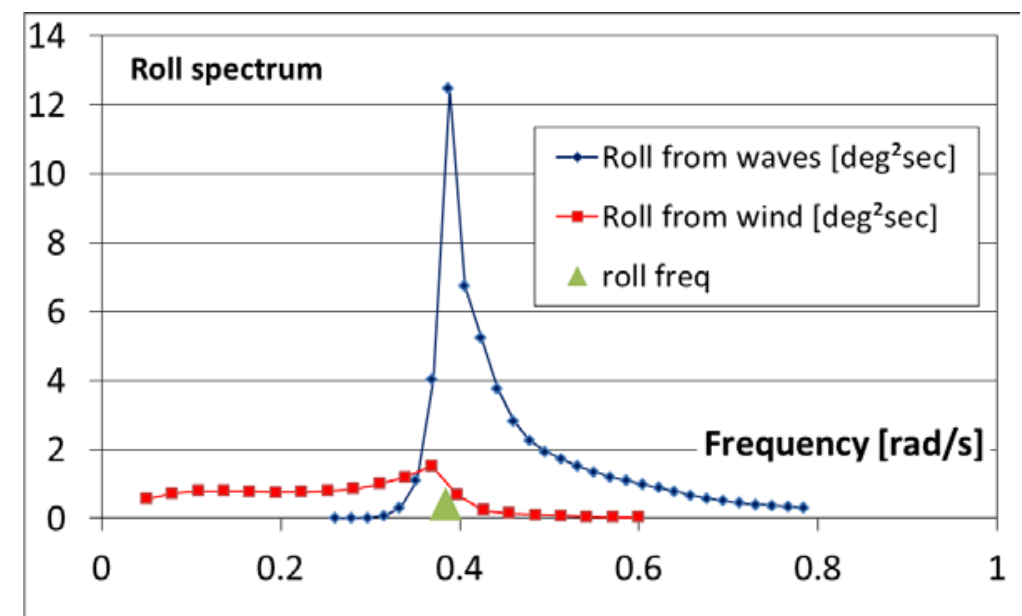

Fig. 6. Linearized roll spectrum, Eq. (12). Target roll angle 37 deg., beam sea condition

\section{Parametric rolling of a ship}

A Panamax container ship with a small forward speed in head sea is considered. The ship parameters and its operational condition are the same as in Jensen et al. (2017) (length $284 \mathrm{~m}$, breadth $32.2 \mathrm{~m}$, draft $10.5 \mathrm{~m}$. Head sea, forward speed $6 \mathrm{~m} / \mathrm{sec}$. in a sea state with a significant wave height $12 \mathrm{~m}$ and wave spectral peak period of $15 \mathrm{sec}$.). The GZ curve for the vessel is shown in Fig. 7, and the discretization of the wave elevation spectrum in Fig. 8.

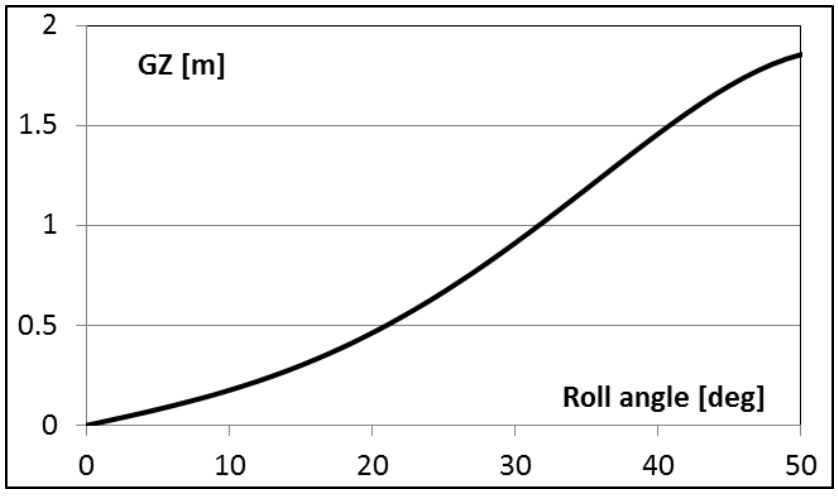

Fig. 7. GZ curve for the vessel, $G M=0.89 m$

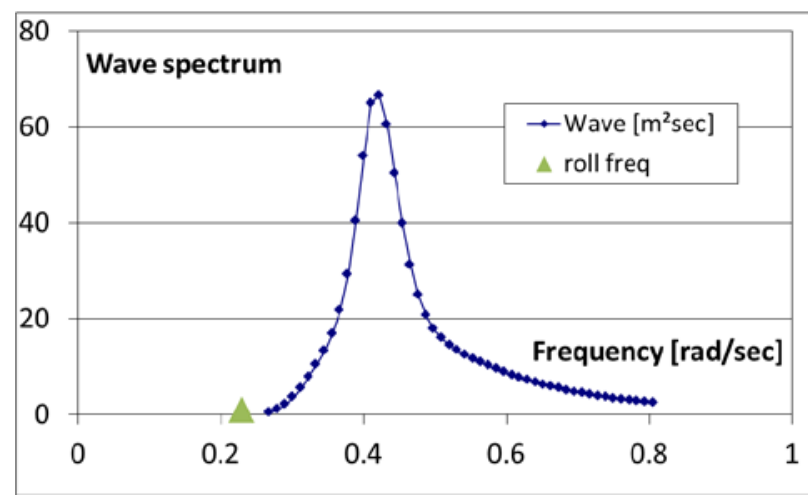

Fig. 8. Wave elevation spectrum

The results from the FORM and Monte Carlo Simulation (MCS) analyses in terms of the reliability index $\beta$ are given in Fig. 9, taken from Jensen et al. (2017). In addition SORM results are included. It is seen that the SORM results are nearly the same as the FORM results indicating that a linearization around the design point is a good approximation, at least within a close range. This finding is contradicting the intuitive conclusion in Jensen et al. (2017). The accuracy of the SORM 
results is very sensitive to the error bound in the FORM calculations, implying a much longer calculation time. In addition the Hessian matrix requires a significant number of calls to the time domain solution procedure. Both a standard second order and a fourth order finite difference approximation have been used to calculate the second derivate of the failure surface at the design point, but with only marginal changes in the SORM results. The main reason for the small fluctuations in the SORM results is therefore probably due to the frequency discretization in the wave elevation spectrum, Fig. 8. Due to these fluctuations SORM results are shown in Fig. 9 as discrete points and not a continuous curve like the FORM results.

The reliability index from the FORM analysis will be used in the extreme value calculations as the difference between FORM and SORM is small and both are conservative compared to the MCS. Overall, the rather good agreement between FORM, SORM and MCS should be noted. Finally, for the slightly higher FORM-2 results given in Jensen et al. (2017), the SORM reliability index is even larger such that the FORM- 2 solution is not a global minimum and is therefore not included in Fig. 9.

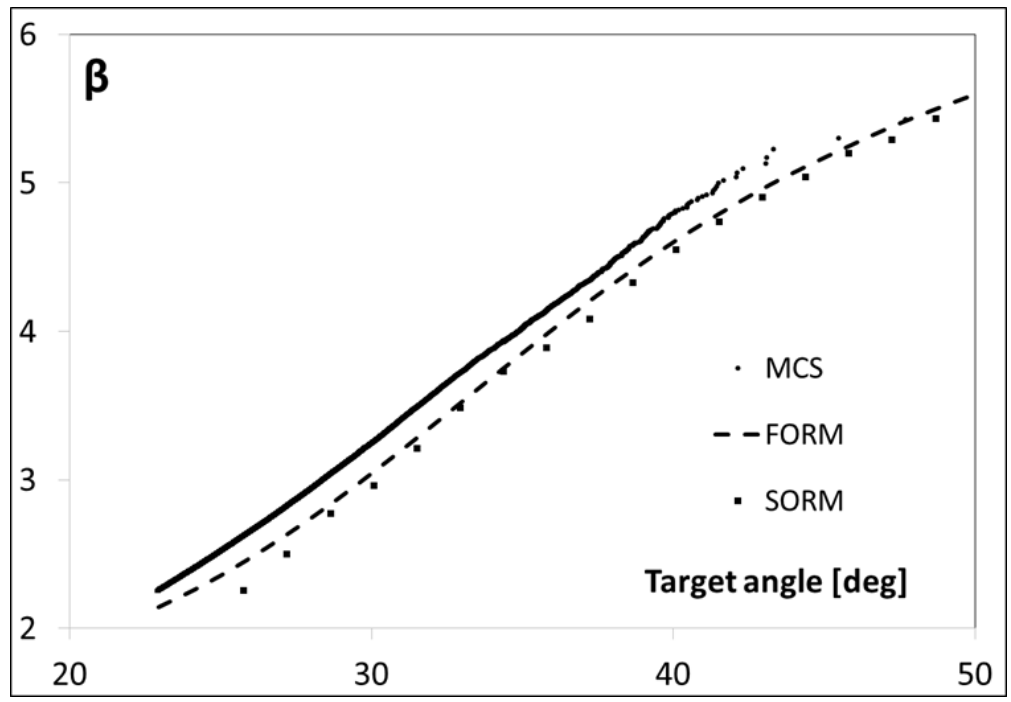

Fig. 9. Reliability index $\beta$ from FORM, SORM and MCS, parametric rolling

In the following the application of the Poisson model, Eq. (23), is discussed. The bandwidth factor $q$, Eq. (20), the Vanmarcke correction factor $c_{v}$, Eq. (19), and the ratio between $T_{0, \text { corr }}$ and $T_{0}$ are shown in Fig. 10. As for the beam sea case $T_{0}$ does not depends significantly on the target angle and thus the variation in $T_{0, \text { corr }}$ with target angle is again mainly due to the Vanmarcke's correction, Eq. (19) and not the FORM linearization. 


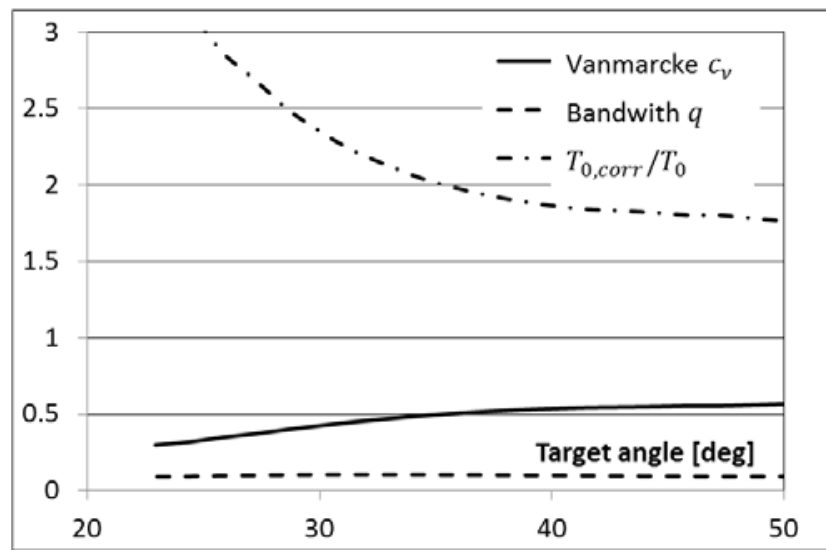

Fig. 10. Bandwidth factor $q$ and Vanmarcke factor $c_{v}$ and the ratio $T_{0, \text { corr }} / T_{0}$, parametric rolling ( $T_{\text {roll }}=27.4 \mathrm{sec}$., $T_{0} \approx 14 \mathrm{sec}$., $T_{0, \text { corr }} \approx 25 \sim 31 \mathrm{sec}$. for $\mathrm{x}_{0}>30 \mathrm{deg}$.)

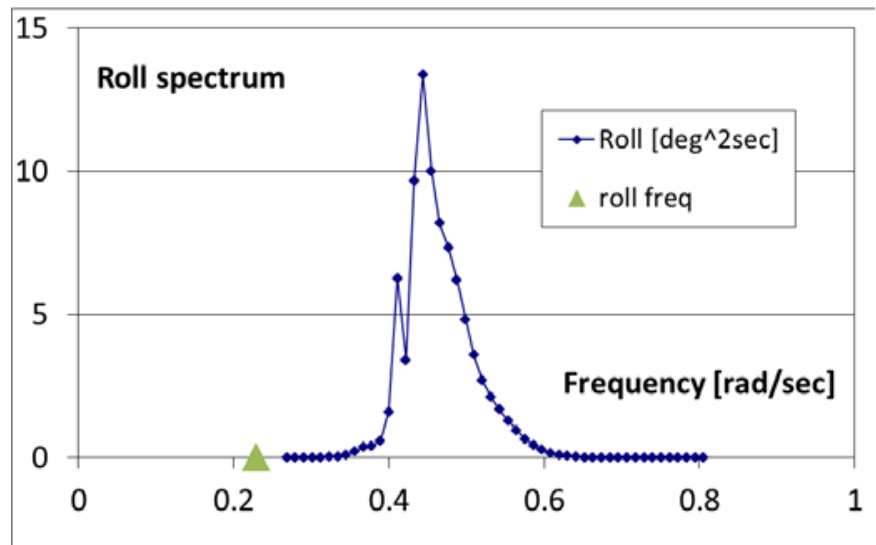

Fig. 11. Linearized roll spectrum, Eq. (12). Target roll angle 33 deg., parametric rolling

Fig. 10 shows that the peaks cannot be considered independent for any roll angles up to at least 50 deg. as the Vanmarcke correction factor $C_{v}$ only is about 0.5 . The reason is that the bandwidth factor $q$ is very low, less than 0.1 , signifying a very narrow-banded response for the FORM linearized roll angle around the design point. An example of the linearized roll spectrum is shown in Fig. 11 for a target roll angle of 33 deg. and it is clearly narrower banded than the one in Fig. 6 for the beam sea rolling case. A possible explanation is that parametric rolling is a pure non-linear process with a very distinct design point, leading to a narrow-banded process able to trigger parametric rolling very precisely. The effect of the very narrow-banded process is reflected in Fig. 10 , where $T_{0, \text { corr }}$ is much larger than the value of uncorrected value $T_{0}$ for roll angles up to 30 
deg. and still nearly twice as large for roll angles above 30 deg., implying that neighbouring peaks are strongly correlated for all roll angles.

The result obtained for the uncorrected value $T_{0}$ is close to mean wave encounter period of 14 sec. and not the roll period $T_{\text {roll }}$ of the vessel $(27.4 \mathrm{sec}$.) as expected. The reason is that parametric rolling is a purely non-linear process implying that the FORM linearization around the design point does not represent the time evolution of the response well even if the reliability index $\beta$ is rather accurate, Fig.9, as compared to MCS. This difference is illustrated in Fig. 12, where the most probable parametric rolling scenario $X\left(t \mid \underline{u}^{*}\right)$ and the linear approximation, Eq. (17), are shown for a target roll angle of $33 \mathrm{deg}$. Clearly the two most probable response curves are very different with respect to average period between peaks where the non-linear result $X\left(t \mid \underline{u}^{*}\right)$ has approximately the same period as the roll period of the vessel and also close to $T_{0, \text { corr }}$. The linearized response based on the roll spectral density in Fig. 12 has on the other hand an average period around $14 \mathrm{sec}$. The slight double peak behaviour in the spectrum is not due to a round-off error in the solution of Eq. (2), but apparently needed to trigger parametric rolling.

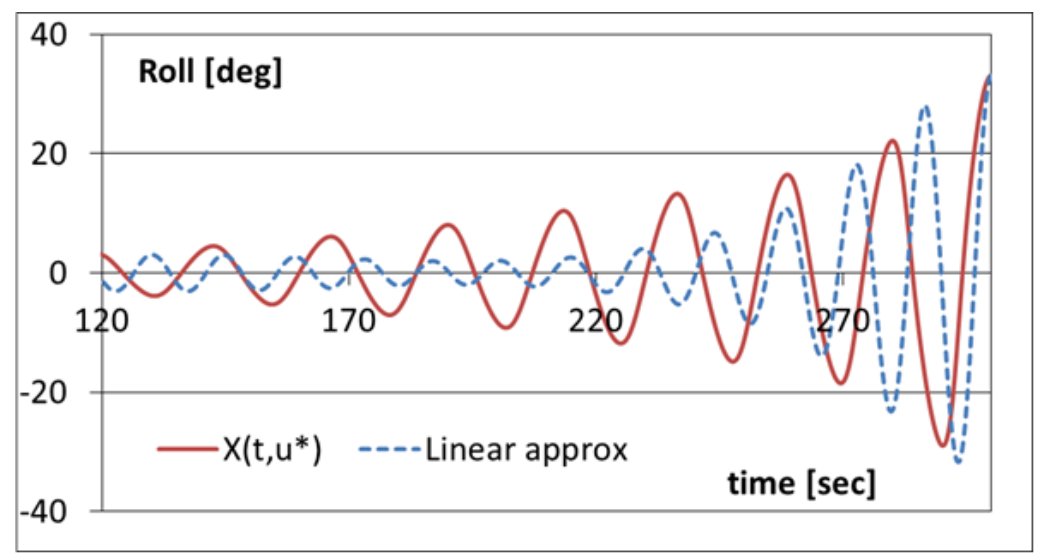

Fig. 12. Most probable parametric rolling scenario $X\left(t \mid \underline{u}^{*}\right)$ and the linear approximation, Eq. (17). Target roll angle 33 deg.

It is somewhat curious that the obvious error in $T_{0}$ (i.e., $14 \mathrm{sec}$.) found using the linearized FORM solution is rather precisely corrected using the Vanmarcke correction for a narrow banded spectrum, yielding an expected mean period close to the mean period seen in the non-linear most probable scenario $X\left(t \mid \underline{u}^{*}\right)$. Further investigation is therefore performed in the next section to see if this holds for other models for parametric roll.

\section{Roll motions of ships with 6-DOF equation}




\section{Equation of motion for parametric rolling}

For verification, an advanced numerical model of the ship motion is applied. The ship motions are defined by $x_{k}$, where $k=1,2, \ldots, 6$ for the surge, sway, heave, roll, pitch and yaw, respectively. The equation of motion for the parametric rolling can be written as

$$
[M]\left\{\ddot{x}_{k}\right\}=\left\{F_{\text {Rad }}\right\}+\left\{F_{\text {Vdamp }}\right\}+\left\{F_{\text {Nonlinear }}\right\}+\left\{F_{\text {Diff }}\right\}+\left\{F_{\text {Ext }}\right\}
$$

The impulse response function approach proposed by Cummins (1962) is used to calculate radiation forces in the time domain and can be expressed as

$$
\begin{gathered}
F_{R a d}(t)=-a_{j k}^{\infty} \ddot{x}_{k}(t)-\int_{-\infty}^{t} K_{j k}(t-\tau) \dot{x}_{k}(\tau) d \tau-c_{j k}^{\infty} ; j=1,2, \ldots, 6 \\
K_{j k}(t)=\frac{2}{\pi} \int_{0}^{\infty}\left(b_{j k}(\omega)-b_{j k}^{\infty}\right) \cos (\omega t) d \omega
\end{gathered}
$$

where $K(t)$ is the impulse response function, $b_{j k}(\omega)$ is the frequency domain solution of the potential damping. $a_{j k}^{\infty}$ and $b_{j k}^{\infty}$ are the infinite frequency added mass and damping, respectively.

$c_{j k}^{\infty}$ is a radiation restoring term, details can be found in e.g., Fonseca and Guedes Soares (1998). The radiation restoring force is time-independent, but forward speed dependent. In this study $b_{j k}^{\infty}$ assumed to be zero. However, it should be noted that in the case of $i \neq j, b_{j k}^{\infty}$ is not zero for forward speed cases and thus recommended to be included for a consistent expression, Ma et al. (2016).

The viscous damping force in roll motion is modeled as

$$
F_{V d a m p, 4}(t)=-\xi_{1} \dot{x}_{4}(t)-\xi_{2} \dot{x}_{4}(t)\left|\dot{x}_{4}(t)\right|-\xi_{3} \dot{x}_{4}^{3}(t)
$$

where, $\xi_{1}, \xi_{2}$ and $\xi_{3}$ are the damping coefficients. For the other modes, no viscous damping is applied. For surge, sway and yaw modes, empirical soft spring forces are added in $\left\{F_{E x t}\right\}$.

For the restoring forces the GZ curve in still water is applied, and the Froude-Krylov $\left\{F_{F k}\right\}$ and the diffraction $\left\{F_{\text {Diff }}\right\}$ forces are converted from the frequency domain solution. In this study, the standard STF method by Salvesen et al. (1970) is used. For the wind forces, the same model as in the previous chapter is applied.

A weakly non-linear approach is applied for the non-linear excitation for parametric rolling. A detailed description of weakly non-linear approaches in connection with the parametric roll phenomenon can be found in Lee and Kim (2017). For computational efficiency, the non-linear excitation forces are calculated using an effective (equivalent) wave concept, Bulian (2008), Kröger (1986), combined with pre-calculated GZ surfaces in regular waves. In the effective wave concept, the irregular sea surface is approximated as a regular wave which has one effective wave length, but varying wave height and crest position. It has been shown previously that calculation 
of parametric roll with the effective wave concept yields results in good agreement with those obtained by direct calculation, Choi et al. (2016).

A post-panamax container ship is chosen for the numerical simulation. The principal particulars and environmental conditions are presented in Table 1. The GZ data for different wave amplitudes and crest positions $x_{c}$ are prepared. Two examples of pre-calculated GZ surfaces are presented in Fig. 13. Here, the effective wave length is chosen to be the ship length, LBP. The damping coefficients in Table 1 are non-dimensionalized as

$$
\xi_{1}^{\prime}=\frac{\xi_{1}}{2 \omega_{\text {roll }}\left(I_{44}+A_{44, \omega_{\text {roll }}}\right)} ; \xi_{2}^{\prime}=\frac{\xi_{2}}{I_{44}+A_{44, \omega_{\text {roll }}}} ; \xi_{3}^{\prime}=\frac{\xi_{3}}{I_{44}+A_{44, \omega_{\text {roll }}}} \omega_{\text {roll }}
$$

The coefficients for the container ship are taken from the model test result for $\mathrm{C} 11$ container ship (performed for 0 and 5 knots, MARIN model 8004-2). For the given speed, the linear coefficient is estimated through linear extrapolation, whereas the quadratic coefficient is assumed constant.

Table 1 Principal particulars and environmental conditions

\begin{tabular}{lc}
\hline Parameters & \\
\hline Vessel & Container ship \\
Length, $\mathrm{L}_{B P}$ & $350.0 \mathrm{~m}$ \\
Breadth, B & $48.2 \mathrm{~m}$ \\
Draught, $\mathrm{T}$ & $15.5 \mathrm{~m}$ \\
Metacentric height, GM ${ }_{\text {sw }}$ & $0.72 \mathrm{~m}$ \\
Roll natural frequency, $\omega_{\text {roll }}$ & $0.14 \mathrm{rad} / \mathrm{sec}$ \\
Non-dimensional damping & $0.029,0.200,0.00$ \\
coefficients, $\xi_{1}^{\prime \prime} \xi_{2}^{\prime \prime}$ and $\xi_{3}^{\prime \prime}$ & $10 \mathrm{knots}$ \\
Forward speed & JONSWAP $(\gamma=3.3)$ \\
Wave spectrum & $3.5 \mathrm{~m}$ \\
Significant height, $\mathrm{H}_{s}$ & $13.1 \mathrm{sec}$ \\
Zero up-crossing period, $\boldsymbol{T}_{\boldsymbol{z}}$ & head sea \\
Wave heading & Davenport \\
Wind spectrum & $26 \mathrm{~m} / \mathrm{sec}$ \\
Mean wind speed, $\boldsymbol{U}_{\text {mean }}$ &
\end{tabular}



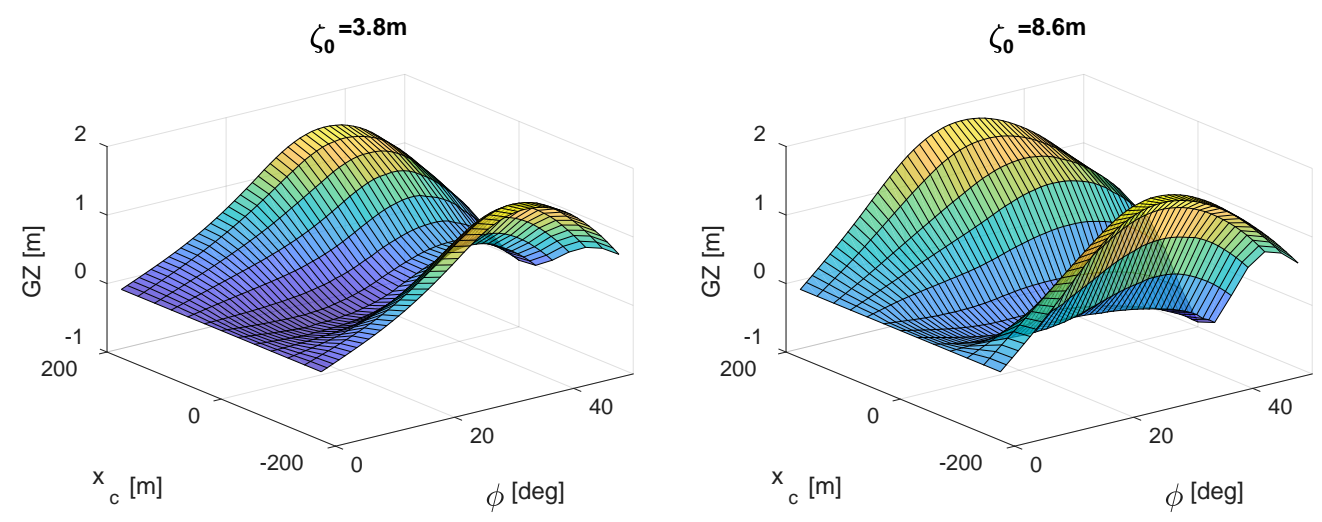

Fig. 13. Pre-calculated GZ data for effective wave amplitude, $\zeta_{0}=3.8 \mathrm{~m}$ (top-left), $\zeta_{0}=8.6 \mathrm{~m}$ (top-right)

\section{FORM results for parametric rolling}

FORM analysis for parametric rolling was performed with the conditions specified in Table 1 . One hundred random variables are used for discretization of waves and the duration time is $1,300 \mathrm{sec}$. The results from the FORM and Monte Carlo Simulation analyses regarding the reliability index $\beta$ are given in Fig. 14. Generally, the very good agreement between FORM and MCS can be seen for roll angles greater than 20 deg. It is noted that SORM results are not included in the plot, as they were found to be significantly larger than the results of FORM and MCS. This is rather contradictory with the results from the 1D model (see Fig. 9), but may show the sensitivity of SORM to the physical modelling applied.

The bandwidth factor $q$ and the Vanmarcke correction factor $c_{v}$ are shown in Fig. 15 (left) and the corresponding values of $T_{0}$ and $T_{0, \text { corr }}$ are presented in Fig. 15 (right). As with the results from the 1D model in Fig. 10, the FORM linearized solution $T_{0}(\approx 20 \mathrm{sec})$ significantly differs from the roll natural period $T_{\text {roll }}(\approx 45 \mathrm{sec})$. However, the result here is that $T_{0, \text { corr }}$ is not close to $T_{\text {roll }}$, but closer to $T_{0}$. This finding implies two important points; 1 ) the Vanmarcke correction factor does not seem to correct the linearized FORM solution in the same way as for the 1D solution. Therefore, 2) as far as the non-linear parametric roll response is concerned, the FORM linearized solution, i.e., $m_{0}$ and $m_{2}$, and the corresponding up-crossing rate in Eq. (15) may lead to a notable error in the extreme value prediction. Therefore, in the next section, the Vanmarcke's factor is calculated in a different way and its applicability to parametric roll will be examined. 


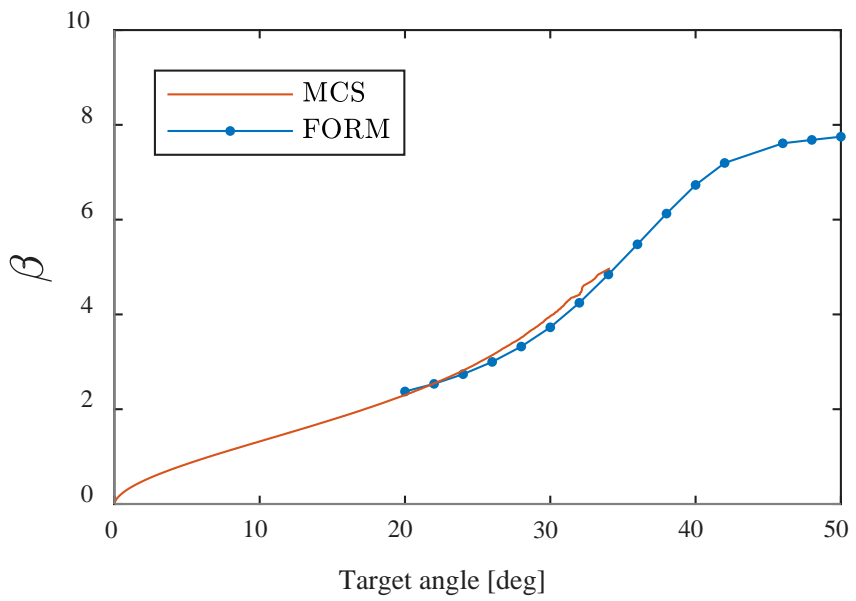

Fig. 14. Reliability index $\beta$ from FORM and MCS ( $3 \times 10^{6}$ simulations) for parametric rolling
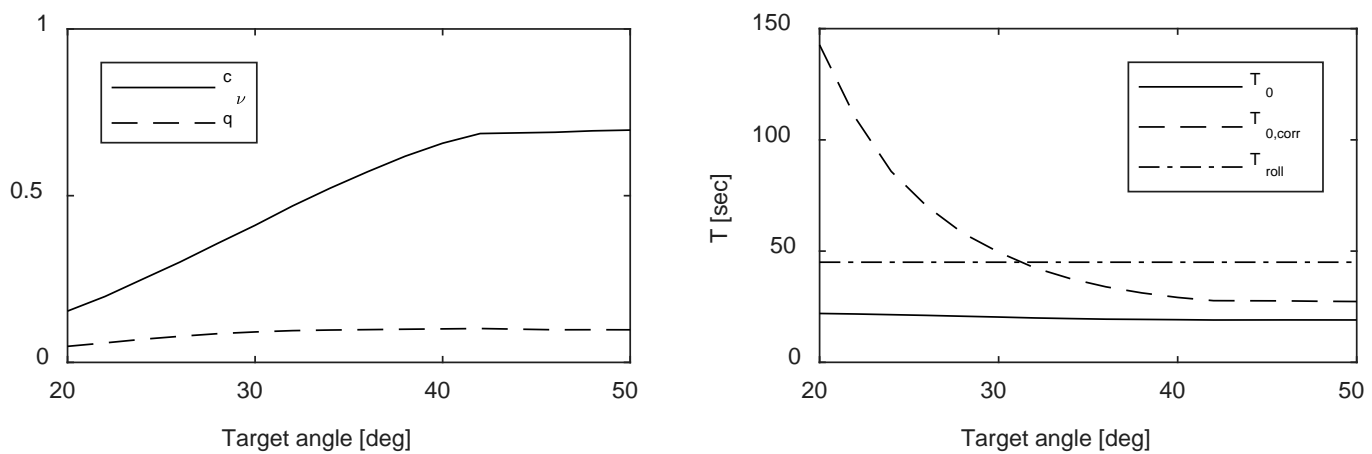

Fig. 15. Bandwidth factor $q$ and Vanmarcke factor $c_{v}$ (left), mean time between up-crossings relative to the mean roll angle (right), parametric rolling

\section{Statistical analysis of parametric rolling}

A simple illustration of narrow-banded response is provided in Fig. 16. The time interval between two adjacent up-crossings of the threshold level can be expressed as $1 / v_{j}^{+}\left(x_{0}\right)$. On the other hand, the time which the response spends under the threshold level is $T_{s a f e, j}$ and its average is defined as $\bar{T}_{\text {safe }}$. 


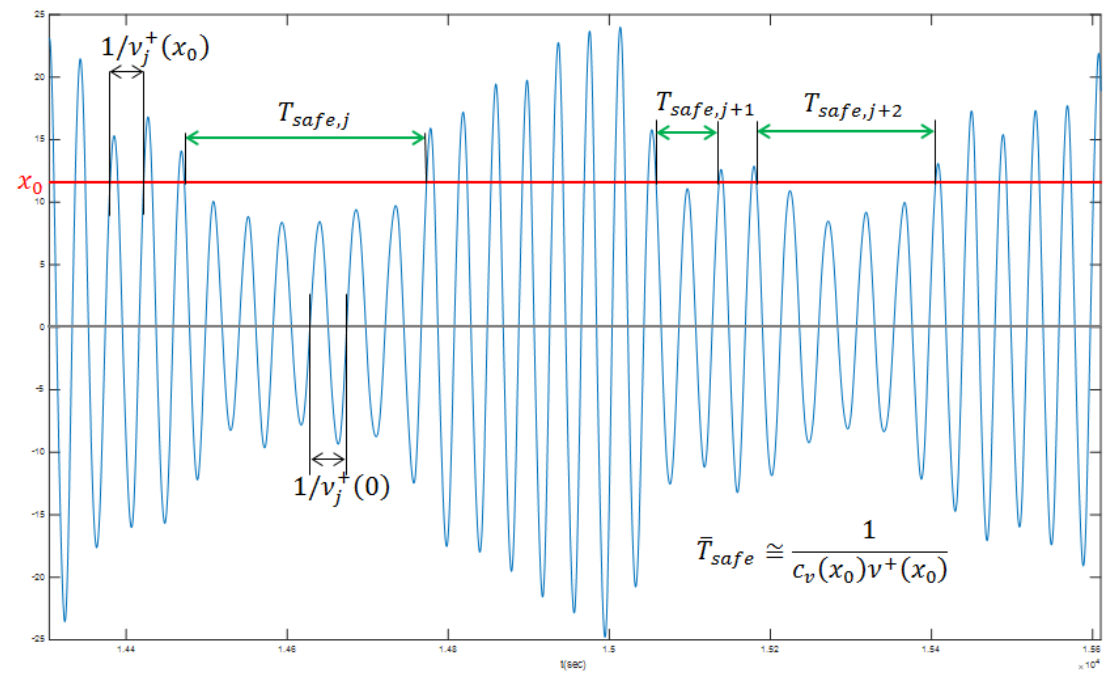

Fig. 16. Illustration of up-crossings and Vanmarcke's factor for a Gaussian process

It is not easy to obtain reliable information using statistical analysis in the case of parametric rolling because the response is non-Gaussian and practically non-ergodic as explained in Belenky et al. (2011) and Bulian et al. (2006). In Fig. 17, probability density functions (PDFs) of the instantaneous roll angle for different realizations are presented. A strong non-linearity can be seen from the shape of PDFs as they do not follow the Gaussian distribution, and moreover different wave realizations produce different distributions. A single temporal analysis with an increased number of wave components and long duration may not be applicable considering computational efficiency. Therefore, Monte-Carlo simulations (MCS) with different random sets (ensembles) are performed to get converged values of the up-crossing rate. The transient stage is identified from the ensemble roll standard deviation as suggested by Bulian et al. (2006), and discarded from the results of the statistical analysis.
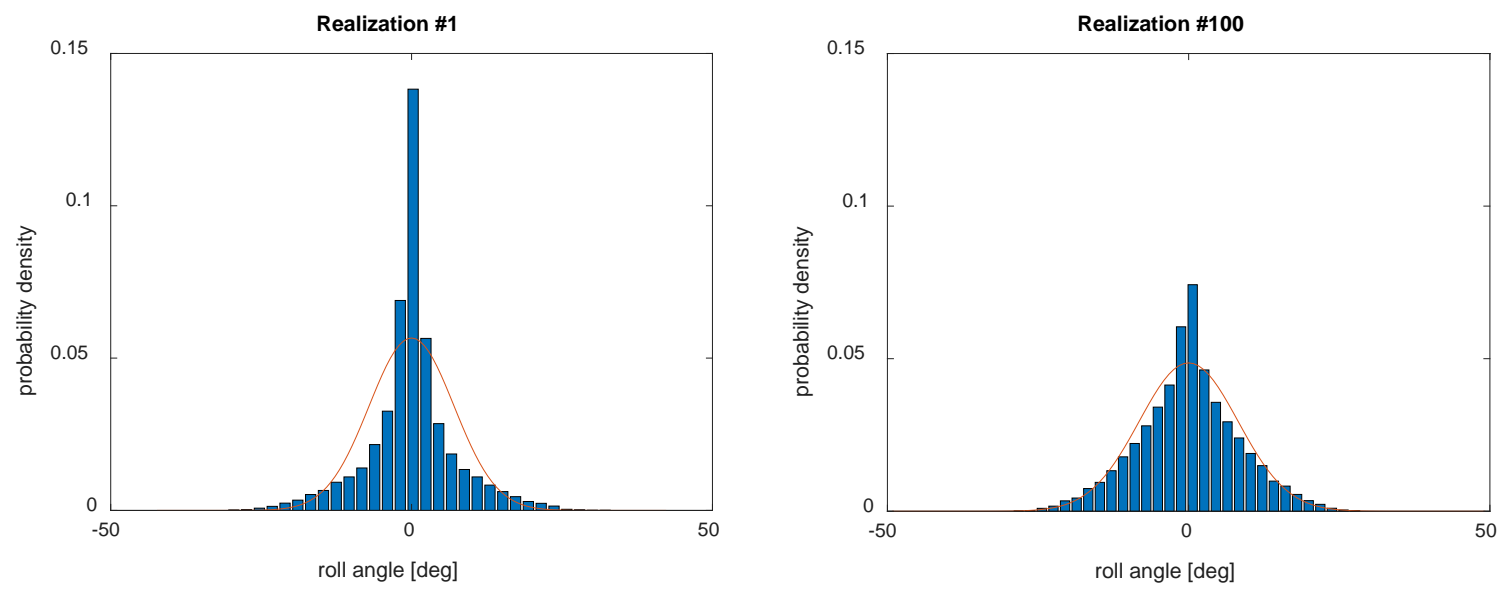

Fig. 17. Probability density function of roll angle in parametric rolling. Red curve is the Gaussian distribution 
The statistical mean up-crossing rate $v_{\text {stat. }}$ is estimated as

$$
\begin{gathered}
v_{\text {stat. }}\left(x_{0}\right)=\sum_{i=1}^{M} n_{i}\left(x_{0}\right) / \sum_{i=1}^{M} T_{i} \\
v_{\text {stat., corr }}\left(x_{0}\right)=c_{v}\left(x_{0}\right) v_{\text {stat. }}\left(x_{0}\right)
\end{gathered}
$$

where, $n_{i}$ is the number of up-crossings during simulation time $T_{i}, \mathrm{M}$ is the total number of simulations. $v_{\text {stat.,corr }}$ is the statistical mean up-crossing rate corrected by Vanmarcke's factor. Additionally, the actual times the response spends under the threshold level $T_{s a f e, j}$ are measured in each signal, hence the up-crossing rate of the mean excursion failure into the unsafe region $v_{\text {stat.unsafe }}$ is calculated by

$$
\begin{gathered}
\bar{T}_{\text {safe }, i}=\sum_{j=1}^{k_{i}} T_{\text {safe }, j} / k_{i} \\
v_{\text {stat.,unsafe }}=M / \sum_{i=1}^{M} \bar{T}_{\text {safe }, i}
\end{gathered}
$$

where, $k_{i}$ is the number of $T_{s a f e, j}$ in $i^{\text {th }}$ realization.

For a Gaussian process, it is expected that

- $v_{\text {Gaussian }} \approx v_{\text {stat. }}$.

- $v_{\text {stat.,unsafe }} \approx v_{\text {stat.,corr }}=c_{v} v_{\text {stat. }}$

where $v_{\text {Gaussian }}$ is the theoretical mean up-crossing rate for a Gaussian response in Eq. (11).

First, the up-crossing rates for Gaussian response in Eq. (11) are compared with the statistical values for two different kind of processes; 1) Irregular wave elevation (Gaussian process), and 2) parametric roll response (strongly non-linear process).

The wave signal is generated from the wave spectrum for parametric rolling in Table. 1 with a duration of $6,000 \mathrm{sec}$. and 15,000 evenly discretized wave components. The frequency range is chosen as a narrow banded case $(0.3 \sim 0.4 \mathrm{rad} / \mathrm{sec}) . M=1,000$ number of simulations is considered for MCS (total simulation time is 1,667 hours). On the other hand, parametric roll responses are obtained from Eq. (24) with 36,000 sec. and 3,000 evenly spaced frequencies. M = 10,000 ensembles are used for MCS (total simulation time is 10,000 hours).

The calculated $c_{v}$ for the wave elevation and parametric roll response are shown in left and right figures in Fig. 18, respectively. $c_{v}$ in both cases tend to increase as the threshold level increases. Notable differences between Fig. 18 (right) and Fig. 15 (left) are seen, even if the ship and the sea condition are the same. This implies that the linearized FORM solution, Fig. 15, is not accurate in this case. 

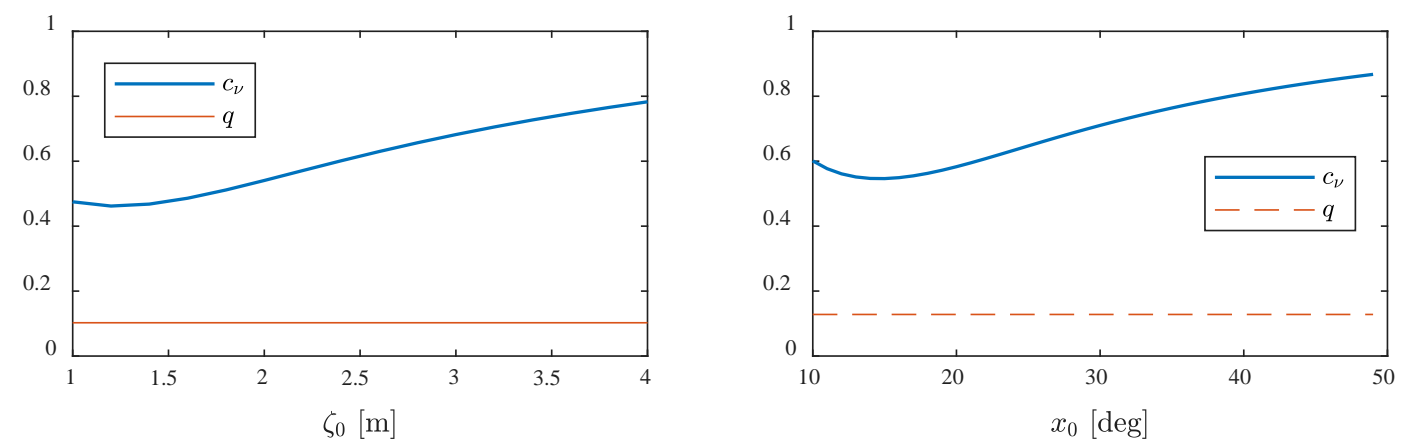

Fig. 18. Bandwidth factor $q$, and Vanmarcke factor $c_{v}$ for wave elevation (left) and for parametric roll (right)

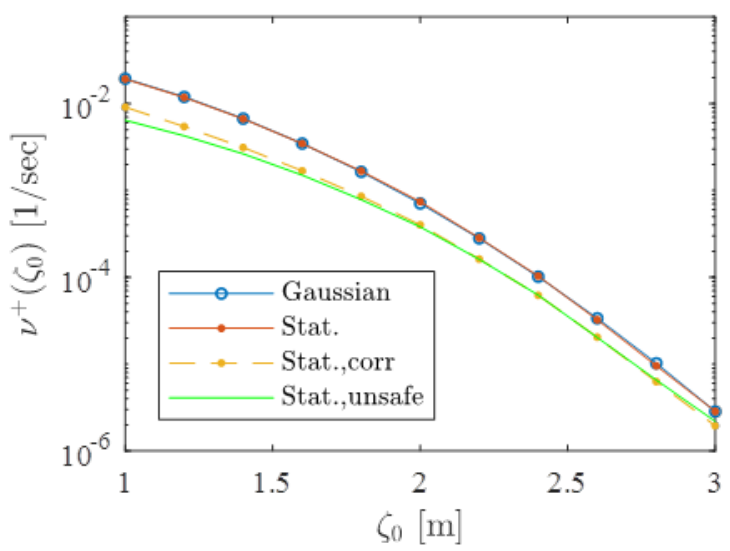

Fig. 19. Comparison of the up-crossing rates for wave elevation

As expected, $v_{\text {Gaussian }}$ and $v_{\text {stat. }}$ are almost identical in the case of wave elevation (Fig. 19). Also the corrected values, $v_{\text {stat,corr }}$ show good agreement with $v_{\text {stat.,unsafe }}$ which is the actual measure of the up-crossing rate for the mean excursion failure, as the wave elevation is Gaussian process. As expected, $v_{\text {Gaussian }}$ ( or $v_{\text {stat. }}$ ) are higher than $v_{\text {stat.,corr }}$ ( or $v_{\text {stat.,unsafe }}$ ), which implies that the Poisson model in Eq. (18) would overestimate the exceedance probability.

The applicability of the Vanmarcke's correction to the nonlinear roll motion is examined through the statistical analysis, considering that the factor is derived under the assumption of a stationary Gaussian process. The ensemble-averaged spectrum is determined from the Monte Carlo Simulations, Belenky and Weems (2012), and its spectral bandwidth, $q$, is calculated by Eq. (20). The corresponding Vanmarcke's factor is then calculated by Eq. (19), where $r=x_{0} / \sqrt{m_{0}}$ instead of $\beta$ is used following the original definition by Vanmarcke (1975). Furthermore, the empirical exponent value, 1.2, for super-clumping effect is neglected.

In Fig. 20 for parametric roll, a significant difference in higher threshold levels between $v_{\text {Gaussian }}$ and $v_{\text {stat. }}$ is seen. This discrepancy is attributed to the strong non-linearity in the parametric roll response and indicates that the use of Eq. (11) for the non-linear process is inadequate.

Fig. 20 also shows that the Vanmarcke correction $v_{\text {stat,corr }}$ is only slightly different to the MCS result $v_{\text {stat.unsafe, }}$ implying that the Vanmarcke correction for the present non-linear problem is a 
good estimate of the up-crossing rate for the mean excursion failure and hence Eq. (21) (or Eq. (18)) becomes valid using $v_{\text {stat, }, \text { corr }}=c_{v} v^{+}\left(x_{0}\right)$ as the corrected up-crossing rate.

Finally, it is seen that $v_{\text {stat.,unsafe }}$ converged to $v_{\text {stat. }}$ for high threshold levels, e.g., after 28 deg. This means that the Vanmarcke's correction is not needed for the extreme roll angles.

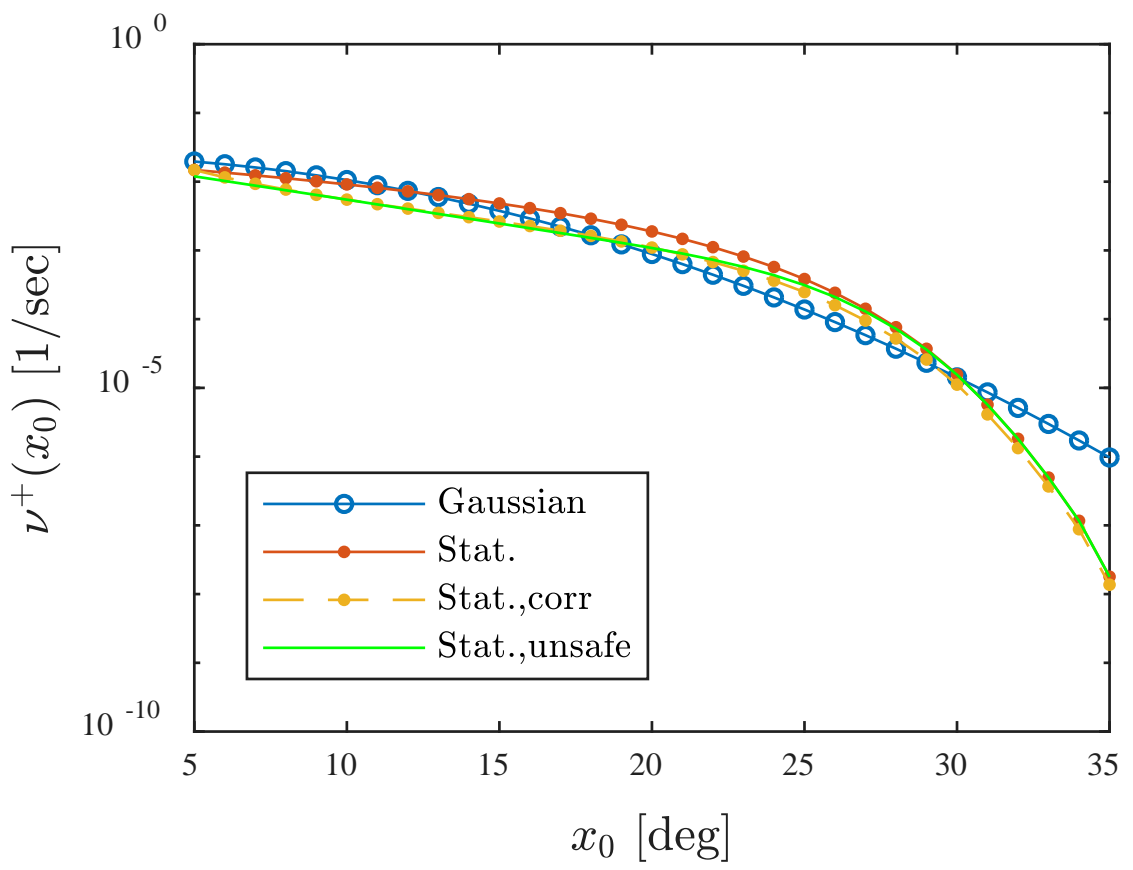

Fig. 20. Comparison of the up-crossing rates for for parametric roll

\section{Conclusion}

The purpose of the paper is to investigate the applicability of the Poisson extreme value model for stationary stochastic processes as applied to non-linear roll motion of ships. The framework is the First Order Reliability Method (FORM), where previous studies have shown that the reliability index $\beta$ obtained by FORM is very close to the results from direct Monte Carlo Simulations (MCS). Hence, the mean period between independent peaks, $T_{0, c o r r}\left(x_{0}\right)$ in the Poisson model, Eq. (23) is focused on. The Vanmarcke's correction is applied to the linearized FORM solution using a simplified 1D model for ship rolling.

Two examples have been considered: ship rolling in beam sea and parametric rolling in head sea. For beam sea a good agreement between the true most probable response and the linearized most probable response for large roll angles (i.e. greater than 30 deg.) is found. Furthermore, there is no significant correlation between peaks. For smaller roll angles adjacent peaks are, however, found to be correlated. 
In the case of parametric rolling the linearized FORM solution, Eq. (11), differs significantly from the true most probable roll response for all target roll angles. The reason is the linearized solution does not capture the bifurcation type of non-linearity in parametric rolling.

In the second part of the paper, a more sophisticated numerical method is applied for further investigations. From the Monte Carlo simulation, it is found that the Vanmarcke's factor can successfully account for the grouping effect for Gaussian process, and provides a slightly unconservative estimation for the present non-Gaussian process, e.g. parametric rolling. It is also found that for the extreme roll peaks $\left(x_{0}>28 \mathrm{deg}.\right)$ the correction is not necessary.

Alternative models for mean up-crossing rate are evaluated for parametric rolling through MonteCarlo simulation in Choi (2018).

\section{Acknowledgments}

The authors would like to thank Prof. Gabriele Bulian for the fruitful discussions. Also Dr.Frans van Walree of MARIN is gratefully acknowledged for making available the roll-decay experimental data for MARIN Model 8004-2.

\section{References}

Belenky, V., Weems, K., 2012. Probabilistic Properties of Parametric Roll - Parametric Resonance in Dynamical Systems, in: Fossen, T.I., Nijmeijer, H. (Eds. ), . Springer New York, New York, NY, pp. 129145.

Belenky, V., Yu, H.-C., Weems, K., 2011. Numerical Procedures and Practical Experience of Assessment of Parametric Roll of Container Carriers - Contemporary Ideas on Ship Stability and Capsizing in Waves, in: Almeida Santos Neves, M., Belenky, V.L., de Kat, J.O., Spyrou, K., Umeda, N. (Eds.), . Springer Netherlands, Dordrecht, pp. 295-305.

Breitung, K., 2015. 40 years FORM: Some new aspects? Probabilistic Eng. Mech. 42, 71- 77.

Breitung, K., 1984. Asymptotic Approximations for Multinormal Integrals. J. Eng. Mech. 110, 357- 366.

Bulian, G., 2008. On an improved Grim effective wave. Ocean Eng. 35, 1811-1825.

Bulian, G., Francescutto, A., Lugni, C., 2006. Theoretical, numerical and experimental study on the problem of ergodicity and "practical ergodicity" with an application to parametric roll in longitudinal long crested irregular sea. Ocean Eng. 33, 1007-1043.

Choi, J.-H., Jensen, J.J., Nielsen, U.D., 2016. A study on the uncertainty and sensitivity in numerical simulation of parametric roll. PRADS 2016 - Proc. 13th Int. Symp. Pract. Des. Ships Other Float. Struct.

Choi, J.H., Jensen, J.J., Kristensen, H.O.H., Nielsen, U.D., Erichsen, H., 2017. Intact stability analysis of dead ship conditions using FORM. J. Sh. Res. 61, 167-176.

Choi, J.H., 2018, Efficient Estimation of Extreme Roll Motion of Ships. PhD thesis, Technical University of Denmark, Dept. Mechanical Engineering, October 2018, 165 pages.

Cummins, W.E., 1962. The impulse response function and ship motions. Schiffstechnik.

Der Kiureghian, A., 2000. Geometry of random vibrations and solutions by FORM and SORM, Probabilistic Engineering Mechanics - PROBABILISTIC ENG MECH.

Fonseca, N., Guedes Soares, C., 1998. Time-Domain Analysis of Large-Amplitude Vertical Ship Motions and Wave Loads. J. Sh. Res. 42, 139- 153.

Fujimura, K., Der Kiureghian, A., 2007. Tail-equivalent linearization method for nonlinear random vibration. Probabilistic Eng. Mech. 22, 63-76. 
Garrè, L., Der Kiureghian, A., 2010. Tail-Equivalent Linearization Method in frequency domain and application to marine structures. Mar. Struct. 23, 322-338.

Jensen, J.J., 2015. Conditional stochastic processes applied to wave load predictions, Weinblum Memorial Lecture 2014. J. Sh. Res. 59, 1-10.

Jensen, J.J., 2011. Extreme value predictions using Monte Carlo simulations with artificially increased load spectrum. Probabilistic Eng. Mech. 26, 399-404.

Jensen, J.J., Capul, J., 2006. Extreme response predictions for jack-up units in second order stochastic waves by FORM. Probabilistic Eng. Mech. 21, 330-337.

Jensen, J.J., Choi, J. hyuck, Nielsen, U.D., 2017. Statistical prediction of parametric roll using FORM. Ocean Eng. 144, 235-242.

Kröger, H.-P., 1986. Rollsimulation von Schiffen im Seegang. Schiffstechnik 33, 187-216.

Köylüoglu H.U., Nielsen, S.R.K. 1994. New approximations for SORM integrals. Structural Safety 13, 235-246.

Lee, J.H., Kim, Y., 2017. Study on added resistance of a ship under parametric roll motion. Ocean Eng. 144, $1-13$.

Ma, S., Wang, R., Zhang, J., Duan, W.Y., Ertekin, R.C., Chen, X.B., 2016. Consistent formulation of ship motions in time- domain simulations by use of the results of the strip theory. Sh. Technol. Res. 63, 146158.

Naess, A., Gaidai, O., Batsevych, O., 2010. Prediction of Extreme Response Statistics of Narrow-Band Random Vibrations. J. Eng. Mech. 136, 290-298.

Salvesen, N., Tuck, E., Faltinsen, O., 1970. Ship motions and sea loads. Trans. SNAME 78, 250-287.

Tompuri, M., Ruponen, P., Forss, M., Lindroth, D., 2015. Application of the Second Generation Intact Stability Criteria in Initial Ship Design, Transactions - Society of Naval Architects and Marine Engineers.

Vanmarcke, E.H., 1975. On the Distribution of the First-Passage Time for Normal Stationary Random Processes. J. Appl. Mech. 42, 215-220. 